\title{
Intestinal Protein Characterisation of SARS-CoV-2 Entry Molecules ACE2 and TMPRSS2 in Inflammatory Bowel Disease (IBD) and Fatal COVID- 19 Infection
}

\author{
Milly J McAllister \\ University of Manchester \\ Kathryn Kirkwood \\ Western General Hospital
}

\section{Shaun C Chuah}

University of Edinburgh MRC Centre for Inflammation Research: The University of Edinburgh Centre for Inflammation Research

\section{Emily J Thompson}

University of Edinburgh MRC Centre for Inflammation Research: The University of Edinburgh Centre for Inflammation Research

\section{Jennifer A Cartwright}

University of Edinburgh MRC Centre for Inflammation Research: The University of Edinburgh Centre for Inflammation Research

\section{Clark D Russell}

University of Edinburgh MRC Centre for Inflammation Research: The University of Edinburgh Centre for Inflammation Research

\section{David A Dorward}

University of Edinburgh MRC Centre for Inflammation Research: The University of Edinburgh Centre for Inflammation Research

\section{Christopher D Lucas}

University of Edinburgh MRC Centre for Inflammation Research: The University of Edinburgh Centre for Inflammation Research

Gwo-tzer Ho ( $\sim$ G.Ho@ed.ac.uk)

University of Edinburgh https://orcid.org/0000-0002-6014-372X

\section{Research Article}

Keywords: COVID19, SARS-CoV-2, IBD, UC, Crohn's disease, inflammation, Gut biology 
DOl: https://doi.org/10.21203/rs.3.rs-495949/v1

License: (c) (1) This work is licensed under a Creative Commons Attribution 4.0 International License. Read Full License

Version of Record: A version of this preprint was published at Inflammation on October 25th, 2021. See the published version at https://doi.org/10.1007/s10753-021-01567-z. 
Intestinal protein characterisation of SARS-CoV-2 entry molecules ACE2 and TMPRSS2 in Inflammatory Bowel Disease (IBD) and fatal COVID-19 infection

Milly J McAllister PhD ${ }^{1}$, Kathryn Kirkwood PhD FRCPath², Shaun C Chuah MBChB ${ }^{1}$, Emily J Thompson $\mathrm{BSC}^{1}$, Jennifer A Cartwright MRCVS ${ }^{1}$, Clark D Russell MBChB ${ }^{1,3}$, David A Dorward PhD ${ }^{1,4}$, Christopher D Lucas PhD ${ }^{1,5}$, Gwo-tzer Ho PhD, FRCP ${ }^{1}$

${ }^{1}$ University of Edinburgh Centre for Inflammation Research, Queen's Medical Research Institute, Edinburgh BioQuarter, 47 Little France Crescent, Edinburgh, EH16 4TJ, U.K.

${ }^{2}$ Pathology Department, Western General Hospital, Crewe Road South, Edinburgh, EH4 2XU, U.K Edinburgh.

${ }^{3}$ Regional Infectious Diseases Unit, Western General Hospital, Crewe Road South, Edinburgh, EH4 $2 X U, U . K$

${ }^{4}$ Department of Pathology, Royal Infirmary of Edinburgh, 51 Little France Crescent, Edinburgh, EH16 4SA, U.K

${ }^{5}$ Department of Respiratory Medicine, Royal Infirmary of Edinburgh, 51 Little France Crescent, Edinburgh, EH16 4SA, U.K

Corresponding author: Dr Gwo-Tzer Ho Edinburgh IBD Science Unit Centre for Inflammation Research University of Edinburgh Scotland, UK

Email: $\quad$ gho@ed.ac.uk 
Abstract: The coronavirus SARS-CoV-2 contributes to morbidity and mortality mainly as a result of immune-pathology in the lungs. Recent data has shown multi-system involvement with widespread viral tropism. Here we present a detailed intestinal protein characterisation of SARS-Cov-2 entry molecules ACE2 and TMPRSS2 in patients with Inflammatory Bowel Disease ([IBD]; Ulcerative Colitis [UC] and Crohn's disease [CD]) with age- and sex-match non-IBD controls; and in those with fatal COVID19 infection. Our study showed that IBD gut inflammation did not influence ACE2 and TRPMSS2 expression. Of interest, colonic protein expression of ACE2 and TRPMSS2 are cytoplasmic distinct to the membranous pattern in the ileum. We observed a significant increase in immune cells within the lamina propria in UC and CD that expressed ACE2 and TRMPSS2 when compared to non-IBD controls. These were identified as plasma cells with multiple myeloma oncogene 1 /interferon regulatory factor 4 (MUM1/IRF4) expression. In six fatal COVID19 cases, there was no gut inflammation despite evidence of viral tropism within the enterocytes. Our data provides evidence for tissue expression of entry molecules ACE2 and TMPRSS2 including a close apposition to plasma cells - both pointing towards a role of the gut in the immune response to SARS-CoV- 2 infection.

Key words: COVID19; SARS-CoV-2; IBD; UC; Crohn's disease; inflammation; Gut biology 
The pathogenic mechanisms of severe acute respiratory syndrome coronavirus 2 (SARS-CoV-2) in mediating the clinical syndrome of COVID19 are increasingly better understood. The SARS-Cov-2 virus gains entry to host cells upon binding of its spike (S) proteins to the angiotensin I converting enzyme 2 (ACE2) receptor where the transmembrane protease (TMPRSS2) primes the $\mathrm{S}$ proteins to facilitate this process ${ }^{1}$. Although mortality and morbidity associated with COVID19 are driven largely by immunopathology in the lung, viral protein and RNA are detected throughout the body ${ }^{2}$ ACE2 and TRMPSS2 for examples, are highly expressed within the gastrointestinal tract, where transcriptomics analysis show highest expressions in the ileal and colonic enterocytes ${ }^{3}$. Diarrhoea is the most common GI symptoms and there is considerable debate whether this is linked to more severe COVID19 (reviewed $\mathrm{in}^{4}$ ). Pertinently, two on-going questions arise on the importance of the gastrointestinal tract and its role in faecal-oral transmission and as a site of host immune response to SARS-CoV-2. Of immediate relevance is the potential importance of local tissue factors such as the presence of chronic inflammation and in common chronic immune-mediated gastrointestinal conditions such as Inflammatory Bowel Diseases (IBD) comprising of Ulcerative Colitis (UC) and Crohn's disease (CD) that affect more than 10 million people globally. In this context, we characterized the gastrointestinal protein expression of ACE2 and TRMPSS2 in individuals with UC and CD; and also in those with fatal COVID19 ( $n=11,10$ and 6 respectively; East of Scotland Ethical Review No 20/ES/0061 and 16/ES/0084 for IBD and COVID19-ICECAP post-mortem studies respectively).

In UC, ACE2 and TMPRSS2 display cytoplasmic expression; whereas in CD, they have a membranous pattern that is more pronounced in the ileum likely due to presence of the brush border in the small bowel (Fig 1A). In UC and CD, ACE2 and TMPRSS2 protein expression levels are similar in actively inflamed and unaffected parts of the colon (Fig 1B and 1C) suggesting that IBD gut inflammation does not lead to an increase of ACE2 and TMPRSS2. In silico analysis of our previously published colonic gene microarray dataset (99 CD, 129 UC and 50 non-IBD controls; Data set available at Gene Expression Omnibus (http://www.ncbi.n/m.nih.gov/geo/ (accessed September 2020) accession: GSE11223 and GSE20881) ${ }^{5}$ support this, by showing no differences in ileo-colonic ACE2 and TMPRSS2 across these groups; and no correlation with inflammation (Fig 1D and 1E). Secondly, we observed a significant increase in immune cells within the lamina propria in UC and CD that expressed ACE2 and TRMPSS2 when compared to non-IBD controls (Fig $\mathbf{1 F}$ and 1G). On histopathological review (GIpathologist, KK), these were identified as plasma cells together with the finding of protein colocalisation with multiple myeloma oncogene 1/interferon regulatory factor 4 (MUM1/IRF4) expression, a commonly used plasma cell marker (Fig $\mathbf{1 H}$ and II). Thirdly, in six fatal COVID19 cases (using tissue generated from the ICECAP post-mortem study), there was no gut inflammation despite evidence of Sars-CoV-2 viral tropism within the enterocytes ${ }^{2}$. Subsequent analysis demonstrates that 
ACE2 and TRMPSS2 enterocyte expressions are not different compared to age- and sex-matched nonIBD controls (Fig 2A and 2B). In line with IBD, we identified MUM1+ plasma cells co-localised with ACE2 within the lamina propria in Covid19 (Fig 2C and 2D).

Gene expression datasets have shown either no upregulation or a trend towards a reduction in ACE2 expression in IBD ${ }^{6-9}$. To date, clinical databases such as SECURE-IBD ${ }^{10}$ have not shown an increase in susceptibility or severity of COVID19 in patients with IBD. Our report which mainly focused on protein (rather than gene) expression data support this. In addition to this, we did not find any evidence of gut inflammation in our cohort of patients with fatal COVID19. Our finding of plasma cells expressing ACE2 in inflamed IBD gut is noteworthy. Recent studies point to the importance of the humoral immune response, particularly IgA neutralizing antibodies to SARS-CoV-2, which is more potent, occur rapidly and remains more persistent. Gaebler et al. show that SARS-CoV-2 antigen persistence in the gut may be the key factor that drives antibody evolution and potency ${ }^{11}$. COVID19 patients have been shown to develop metabolically hyperactive plasmablasts during inflammatory states ${ }^{12}$. IgA antibodies dominated the early SARS-CoV-2-specific antibody response compared with IgG and IgM and was associated with expansion of IgA plasmablasts with mucosal homing characteristics ${ }^{13}$. Although our primary data does not show gut inflammation as a specific factor for SARS-CoV-2 entry mechanism, the high expression of ACE2 and TMPRSS2 along with the apposition of plasma cells in the gut lamina propria suggest that the gastrointestinal tract may play a role in SARS-CoV-2 entry and host humoral immune response in COVID19.

Contributions: All authors contributed to the study conception and design led by GTH. MJM performed all immunohistochemistry experiments and carried out primary analyses. KK carried out histopathological interpretation and curated IBD gut specimens. SCC and JAC contributed to manuscript preparation and data analyses. CDR, CDL and DAD provided Covid19 gut section and all relevant clinical data and interpretation. EJT carried out microarray data analyses. K All authors read and approved the final manuscript. GTH and MJM wrote the manuscript.

\section{Competing interest: None.}

Ethical approval: East of Scotland Research Ethical Approval No 20/ES/0061 and No 16/ES/0084

\section{Funding acknowledgement:}

Gut Immunobiology Fund to G.T.H. Inflammation in COVID-19: Exploration of Critical Aspects of Pathogenesis (ICECAP) receives funding and support from the Chief Scientist Office (COV/EDI/20/10" to D.A.D., C.D.L. and 
C.D.R) LifeArc (through the University of Edinburgh STOPCOVID funding award to D.A.D., and C.D.L.), UK Research and Innovation (UKRI) (Coronavirus Disease [COVID-19] Rapid Response Initiative; MR/V028790/1 to C.D.L. and D.A.D.) and Medical Research Scotland (CVG-1722-2020 to D.A.D., C.D.L. and C.D.R.). C.D.L. is funded by a Wellcome Trust Clinical Career Development Fellowship (206566/Z/17/Z). C.D.R. is supported by the Medical Research Council (grant MC_PC_19059) as part of the International Severe Acute Respiratory Infection Consortium Coronavirus Clinical Characterisation Consortium (ISARIC-4C).

Consent to Participate: All human subjects have consented via ethical approvals above.

Consent for Publication: All human subjects have consented for publication of research data via ethical approvals above.

Acknowledgements: Not applicable.

Availability of data and materials: Full data presented in this data is available on request to corresponding author. 


\section{Supplementary:}

\section{Methodology}

\section{Patient cohorts}

Intestinal resection specimens from 21 patients with Inflammatory Bowel Disease (Crohn's Disease $n=11$ [ileum $n=3$, colon $n=9$ ], Ulcerative Colitis $n=10$ [colon $n=10$ ]) and 10 non-IBD control patients (colon resection for colorectal cancer) were studied. All surgical resections were carried out between $1^{\text {st }}$ Jan 2016 and $31^{\text {st }}$ December 2020 in the Western General Hospital, Edinburgh (East of Scotland Research Ethical Approval No 20/ES/0061). The groups were matched for age- and gender; mean age 66,64 and 68 years old for UC, CD and non-IBD controls at time of resection respectively. Histological analysis of inflamed vs. non-inflamed areas in IBD resection specimens were carried out by GI pathologist (KK).

Additionally, enteric tissue samples were obtained from post-mortem examinations conducted on six patients with pre-mortem PCR-confirmed SARS-CoV-2 infection a median of 19.6 hours (IQR 13.6-47.2) after death, as previously reported ${ }^{2}$ (East of Scotland Research Ethical Approval No 16/ES/0084). Formalin-fixed paraffin-embedded (FFPE) tissue blocks were prepared following standard processes. Patients with Covid-19 were all male and had a mean age of 71 years. None of the patients had preexisting intestinal disease. All patients had hypoxic respiratory failure with radiological evidence of viral pneumonitis. SARS-CoV-2 was present in colon tissue from all six patients (detected using multiplex PCR using ARTIC Network protocol, confirmed by sequencing, as described with subgenomic messenger RNA detected in 5/6, indicative of viral RNA synthesis.

\section{Immunohistochemistry}

Formalin-fixed paraffin-embedded (FFPE) tissues biopsies were processed into $4 \mu \mathrm{m}$ sections and stained for haematoxylin and eosin. Sections were de-paraffinised in xylene, rehydrated through graded alcohol, and heated for 5 minutes under pressure for antigen retrieval in Citrate buffer pH6 ( $2 \mathrm{mM}$ Sodium Citrate and 8mM Citric Acid). Endogenous peroxidase activity was blocked using 3\% $\mathrm{H}_{2} \mathrm{O}_{2}$ for 30 minutes and non-specific background staining was blocked for 30 minutes in $10 \%$ horse serum in Tris-buffered saline (TBS). Primary antibodies for ACE2 (Cat Ab15348; Abcam) and TMPRSS2 (Cat Ab92323; Abcam) were incubated overnight at $4^{\circ} \mathrm{C}$ diluted at $1: 1000$. Sections were incubated in ImmPRESS detection kit (Vector Laboratories, UK) and visualised using 3,3-diaminobenzidine tetrahydrochloride (DAB, Dako, UK). Samples were then counterstained, dehydrated, and mounted. Antibody specificity for ACE2 and TMPRSS2 was confirmed using an IgG isotype control (Cat Ab172730; Abcam). Slides were scanned using Zeiss AxioScanner (Zeiss, Germany) and analysed using Carl Zeiss 
Zen 2.6 Blue edition programme (Zeiss, Germany). Staining intensity for ACE2 and TMPRSS2 was performed using a weighted histo-score method for cytoplasmic and membrane expression in the epithelia and lamina propria. In brief, the score was calculated by sum of $(1 \mathrm{X} \%$ cells staining weakly positive $)+(2 \times \%$ cells staining moderately positive $)+(3 \times \%$ cells staining strongly positive $)$ with a maximum of 300 ( $100 \%$ strongly stained) and a minimum of 0 (100\% with no staining). The percentage of ACE2 or TMPRSS2 positive immune cells within the lamina propria was calculated by counting the percentage of positive cells within three representative $0.6 \mathrm{~mm} \times 0.6 \mathrm{~mm}$ areas. ACE2 and TMPRSS2 staining intensity and positive immune cell counts were scored by two independent observers.

\section{Immunofluorescence}

Tissue sections were de-paraffinised and rehydrated before undergoing antigen retrieval in Citrate Buffer pH6. Samples were blocked for 1 hour using $2 \%$ fetal calf serum and incubated in primary antibodies ACE2 (1:1000, Cat Ab15348; Abcam) and Anti-MUM1 (1:100, Cat Ab247079; Abcam) overnight at $4^{\circ} \mathrm{C}$ and for 1 hour at room temperature receptively. Sections were incubated in Alexa Fluor $^{\circledR} 488$ (Cat Ab150077; Abcam) and Alexa Fluor ${ }^{\circledR} 555$ (Cat Ab150078; Abcam) receptively and visualised using Zeiss LSM 780 Confocal followed by analysis on Carl Zeiss ZEN 2 blue edition software (Zeiss, Germany). Staining was categorised based on absence and/ or presence of ACE2 and/ or MUM1, analysed in three representative $0.6 \mathrm{~mm} \times 0.6 \mathrm{~mm}$ areas within the lamina propria, and scored by two independent observers.

\section{Gene microarray}

Gene expression for ACE2 and TRMPSS2 were analysed from gut biopsies taken from 99 Crohn's Disease, 129 Ulcerative Colitis and 50 non-IBD control patients. Full details of tissue acquisition and processing are previously detailed ${ }^{5}$. The whole data set is available at Gene Expression Omnibus (http://www.ncbi.nlm.nih.gov/geo/ (accessed September 2020) accession: GSE11223 and GSE20881.

\section{Statistics}

Statistical analysis was performed using SPSS Version 25.0 (IBM, USA) and GraphPad Prism Version 5 (GraphPad Software, USA) for Mann-Whitney U-Test. Interclass correlation coefficients were employed to confirm two independent scores of tissues staining intensity. 


\section{References:}

1. Hoffmann, M., et al. SARS-CoV-2 Cell Entry Depends on ACE2 and TMPRSS2 and Is Blocked by a Clinically Proven Protease Inhibitor. Cell 181, 271-280.e278 (2020).

2. Dorward, D.A., et al. Tissue-Specific Immunopathology in Fatal COVID-19. Am J Respir Crit Care Med 203, 192-201 (2021).

3. Zhang, H., et al. Digestive system is a potential route of COVID-19: an analysis of single-cell coexpression pattern of key proteins in viral entry process. Gut 69, 1010-1018 (2020).

4. Guo, M., Tao, W., Flavell, R.A. \& Zhu, S. Potential intestinal infection and faecal-oral transmission of SARS-CoV-2. Nat Rev Gastroenterol Hepatol (2021).

5. Noble, C.L., et al. Regional variation in gene expression in the healthy colon is dysregulated in ulcerative colitis. Gut 57, 1398-1405 (2008).

6. Nowak, J.K., et al. Age, Inflammation, and Disease Location Are Critical Determinants of Intestinal Expression of SARS-CoV-2 Receptor ACE2 and TMPRSS2 in Inflammatory Bowel Disease. Gastroenterology 159, 1151-1154 e1152 (2020).

7. Burgueno, J.F., et al. Expression of SARS-CoV-2 Entry Molecules ACE2 and TMPRSS2 in the Gut of Patients With IBD. Inflamm Bowel Dis 26, 797-808 (2020).

8. Suarez-Farinas, M., et al. Intestinal Inflammation Modulates the Expression of ACE2 and TMPRSS2 and Potentially Overlaps With the Pathogenesis of SARS-CoV-2-related Disease. Gastroenterology 160, 287-301 e220 (2021).

9. Verstockt, B., et al. Intestinal receptor of SARS-CoV-2 in inflamed IBD tissue seems downregulated by HNF4A in ileum and upregulated by interferon regulating factors in colon. J Crohns Colitis (2020).

10. Gajendran, M., et al. Inflammatory bowel disease amid the COVID-19 pandemic: impact, management strategies, and lessons learned. Ann Gastroenterol 33, 591-602 (2020).

11. Gaebler, C., et al. Evolution of antibody immunity to SARS-CoV-2. Nature (2021).

12. Bernardes, J.P., et al. Longitudinal Multi-omics Analyses Identify Responses of Megakaryocytes, Erythroid Cells, and Plasmablasts as Hallmarks of Severe COVID-19. Immunity 53, 1296-1314 e1299 (2020).

13. Sterlin, D., et al. IgA dominates the early neutralizing antibody response to SARS-CoV-2. Sci Transl Med 13(2021). 


\section{Figure Legends:}

Figure 1: ACE2 and TMPRSS2 protein and gene expression in Ulcerative Colitis (UC), and Crohn's Disease (CD) patients and non-IBD controls. A: Immunohistochemistry of ACE2 and TMPRSS2 protein expression within the cytoplasm (C) and membrane (M) of non-IBD ( $n=10), U C$ (colon) ( $n=9)$, and CD (ileum and colon) ( $n=11$ ) patients. B: ACE2 protein weighted histoscore expression within the cytoplasm (C) or membrane (M) of uninflamed $(n=8)$ or inflamed $(n=7)$ colonic tissue. C: TMPRSS2 protein weighted histoscore expression within the cytoplasm (C) or membrane (M) of uninflamed $(n=8)$ or inflamed $(n=7)$ colonic tissue. $D$ : ACE2 gene expression in uninflamed ( $n=57)$ vs. inflamed $(n=67)$ colonic UC vs non-IBD controls ( $n=50)$. E: TMPRSS2 gene expression in uninflamed $(n=57)$ vs. inflamed ( $n=67)$ colonic UC vs non-IBD controls ( $n=50)$. F: ACE2 positive cells (\%) within lamina propria in inflamed and uninflamed colon of IBD vs. non-IBD controls ( ${ }^{* *} p<0.001$ and $\left.{ }^{* * *} p<0.0001\right)$. G: TMPRSS2 positive cells (\%) within lamina propria in inflamed and uninflamed colon of IBD vs. non-IBD controls ( $p=N S$ ). H: ACE2 expressing cells within the lamina propria of uninflamed and inflamed IBD colon. I: Immunofluorescence co-staining of ACE2 and MUM1 (plasma cell marker) within the lamina propria of colonic IBD. Error bars represent standard error of mean (SEM). DAPI was included as a nuclear stain and scale bars represent $20 \mu \mathrm{m}$.

Figure 2: ACE2, TMPRSS2 and MUM1 protein expression in fatal COVID19 (SARS-CoV-2 PCR positive) ileum and colon sections; and non-IBD controls. A: ACE2 protein weighted histoscore expression of cytoplasm (C) or membrane (M) of COVID19 ileum or colon versus non-IBD controls. B: TMPRSS2 protein weighted histoscore expression of cytoplasm (C) or membrane (M) of COVID19 ileum or colon versus non-IBD controls. C: Immunohistochemistry of ACE2 and TMPRSS2 protein expression within the ileum and colon of COVID-19. D Immunofluorescence co-staining of ACE2 and MUM1 (plasma cell marker) within the lamina propria of COVID-19 positive ileal tissue. Error bars represent standard error of mean (SEM). DAPI was included as a nuclear stain and scale bars represent $20 \mu \mathrm{m}$. 

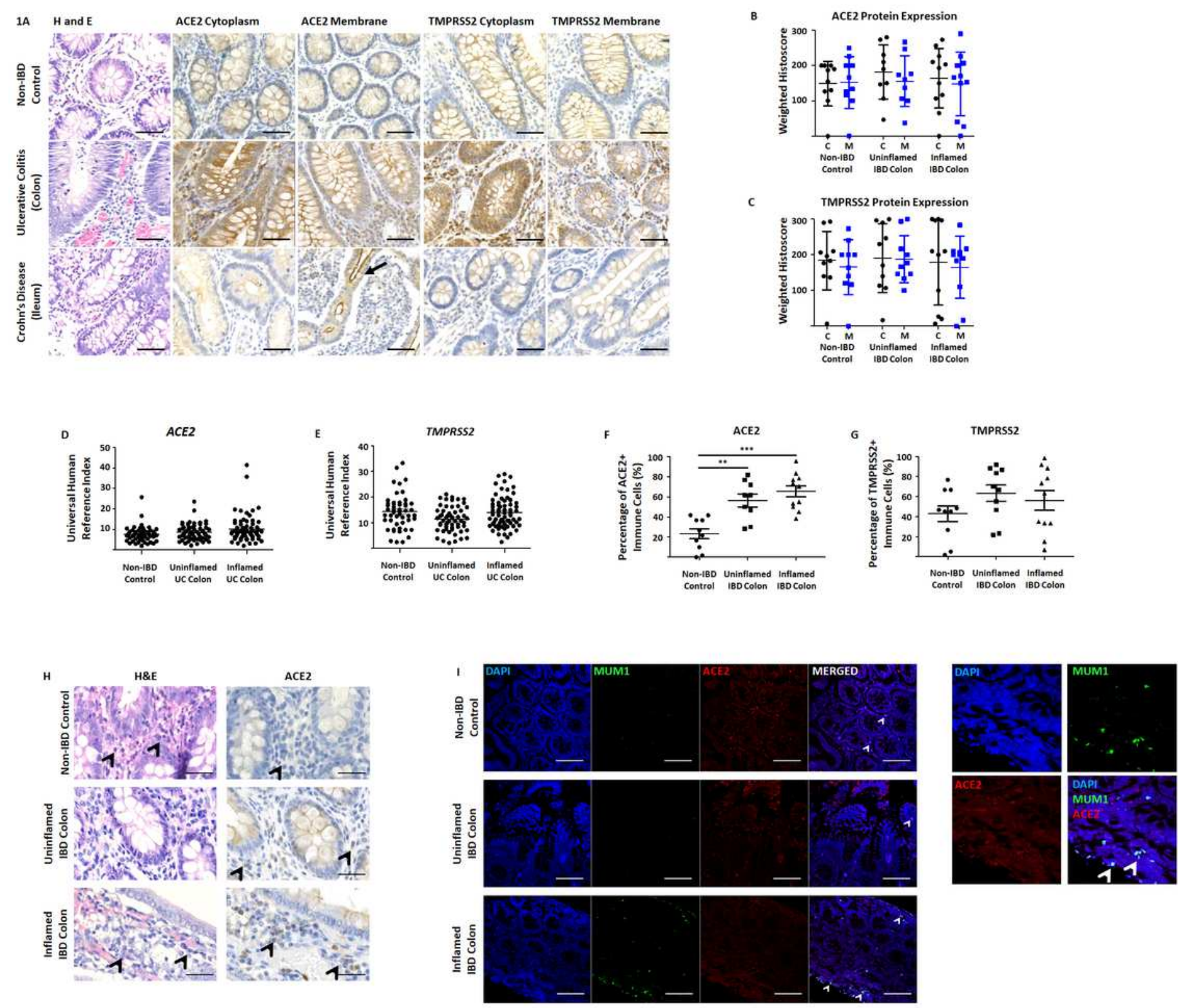

\section{Figure 1}

ACE2 and TMPRSS2 protein and gene expression in Ulcerative Colitis (UC), and Crohn's Disease (CD) patients and non-IBD controls. A: Immunohistochemistry of ACE2 and TMPRSS2 protein expression within the cytoplasm (C) and membrane (M) of non-IBD ( $n=10), U C$ (colon) ( $n=9)$, and CD (ileum and colon) $(n=11)$ patients. B: ACE2 protein weighted histoscore expression within the cytoplasm (C) or membrane $(M)$ of uninflamed $(n=8)$ or inflamed $(n=7)$ colonic tissue. C: TMPRSS2 protein weighted histoscore expression within the cytoplasm (C) or membrane (M) of uninflamed $(n=8)$ or inflamed $(n=7)$ colonic tissue. D: ACE2 gene expression in uninflamed $(n=57)$ vs. inflamed $(n=67)$ colonic UC vs non-IBD controls ( $n=50)$. E: TMPRSS2 gene expression in uninflamed $(n=57)$ vs. inflamed $(n=67)$ colonic UC vs non-IBD controls $(n=50)$. F: ACE2 positive cells $(\%)$ within lamina propria in inflamed and uninflamed 
colon of IBD vs. non-IBD controls (** $p<0.001$ and $* \star \star ~ p<0.0001)$. G: TMPRSS2 positive cells (\%) within lamina propria in inflamed and uninflamed colon of IBD vs. non-IBD controls $(p=N S)$. H: ACE2 expressing cells within the lamina propria of uninflamed and inflamed IBD colon. I: Immunofluorescence co-staining of ACE2 and MUM1 (plasma cell marker) within the lamina propria of colonic IBD. Error bars represent standard error of mean (SEM). DAPI was included as a nuclear stain and scale bars represent $20 \mu \mathrm{m}$.
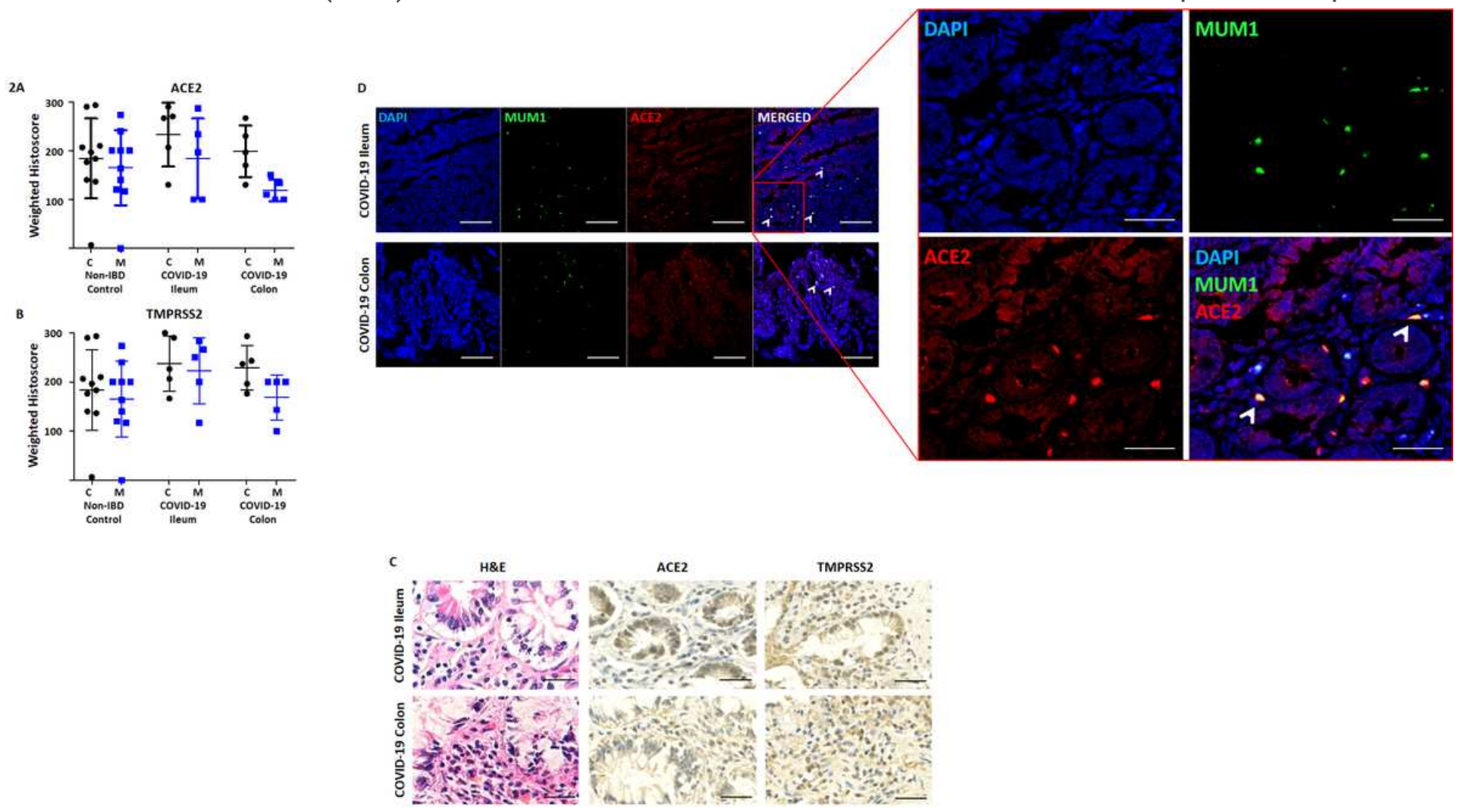

\section{Figure 2}

ACE2, TMPRSS2 and MUM1 protein expression in fatal COVID19 (SARS-CoV-2 PCR positive) ileum and colon sections; and non-IBD controls. A: ACE2 protein weighted histoscore expression of cytoplasm (C) or membrane (M) of COVID19 ileum or colon versus non-IBD controls. B: TMPRSS2 protein weighted histoscore expression of cytoplasm (C) or membrane (M) of COVID19 ileum or colon versus non-IBD controls. C: Immunohistochemistry of ACE2 and TMPRSS2 protein expression within the ileum and colon of COVID-19. D Immunofluorescence co-staining of ACE2 and MUM1 (plasma cell marker) within the lamina propria of COVID-19 positive ileal tissue. Error bars represent standard error of mean (SEM). DAPI was included as a nuclear stain and scale bars represent $20 \mu \mathrm{m}$. 